\title{
Single-cell RNA sequencing reveals B cell-T cell interactions in vascular adventitia of hyperhomocysteinemia-accelerated atherosclerosis
}

\section{Dear Editor}

Cardiovascular disease (CVD) is the leading cause of death around the world (Truelsen, et al., 2015). Atherosclerosis, the dominant underlying cause of CVD, is a chronic inflammatory disease characterized by lipid accumulation and immune cell infiltration in plaques and vessels (Weber, et al., 1950). The immune microenvironment is critical for the development of atherosclerosis. Homocysteine (Hcy) is an intermediate product of methionine metabolism, and its elevation in plasma (>15 $\mu \mathrm{mol} / \mathrm{L})$, known as hyperhomocysteinemia (HHcy), is an independent risk factor for atherosclerosis. HHcy is more common in Asia because of genetic factors and dietary habits (Huo, et al., 2015). folic acid supplement is one of the most important way to treat $\mathrm{HHcy}$ in clinic. Although HHcy potentiates atherosclerosis mainly through endothelial injury and inflammatory activation (Luo, et al., 2016), a comprehensive understanding of the immune microenvironment and potential mechanisms in HHcy-accelerated atherosclerotic aortas (HHcy-AA) is still lacking.

With the development and application of single-cell RNA sequencing (scRNA-seq), elucidation of complex cellular compositions and phenotypic heterogeneity of vascular immune cells has recently become possible. Firstly, we established a HHcy mouse model that has been proven to successfully induce inflammatory cell infiltration and aggravate atherosclerosis (Dai, et al., 2006). Female $\mathrm{ApoE}^{-/-}$mice were fed a normal chow diet (CD) with drinking water supplemented with or without $1.8 \mathrm{~g} / \mathrm{L}$ Hcy for 4 weeks. Western diet (WD)-fed $\mathrm{ApoE}^{-/-}$mice were also included as an additional group. Single live CD45 ${ }^{+}$leukocytes were then sorted from isolated aortas (without adipose tissue) from three groups by fluorescence-activated cell sorting (FACS) and subjected to scRNA-seq (Fig. S1A and S1B). The concentration of Hcy in plasma was successfully increased by Hcy administration in the HHcy $\mathrm{ApoE}^{-/-}$group (Fig. S1C). Oil Red $O$ staining of aortic roots showed significantly increased plaque formation in $\mathrm{HHcy}_{\mathrm{ApoE}}^{-/-}$mice and WD $\mathrm{ApoE}^{-/-}$ mice, confirming the aggravation of atherosclerosis by HHcy and WD (Fig. S1D). After a standard preprocessing workflow and exclusion of nonimmune cells with the $\mathrm{R}$ package Seurat (Stuart, et al., 2019), we obtained a total of 20,309 cells for further analysis (Fig. S1E-G).

Unsupervised graph-based clustering revealed 15 immune cell clusters (Fig. S2A and S2B), as shown in the t-distributed stochastic neighbor embedding ( $t-S N E$ ) plot (Fig. 1A). All identified cell clusters were found in three groups and can be grouped as six immune cell types, including B cells (Cd79a, C79b, and Cd19), macrophages (Adgre1, Mrc1, and Retnla), neutrophils (Cxcr2 and Lcn2), T cells (Cd3e, Cd4, and Cd8a), DCs (Cd209a and Flt3), and mast cells (Cpa3, Mcpt4 and Mrgprb1). In addition, cluster 9 was a cluster of mixed proliferating cells (Mki67) (Figs. 1A and S2B-I). B cells and macrophages represented two major cell types in our scRNA-seq data. Four B cell clusters (clusters $0,3,8,12$ ) were detected, among which cluster 0 (named as "B cells_1") displayed higher $\mathrm{H} 2-\mathrm{Oa}$ and $\mathrm{Cr} 2$ expression (Fig. S3A and S3B), indicating the function of antigen presentation. Spib and Ms4a1 were highly expressed in cluster 8 (Fig. S3C and S3D), suggesting that this population may be memory B cells. Cluster 12 had higher expression of Ssr4 and Xbp1, which were the markers of plasma cells (Fig. S3E and S3F). Cluster 3 (B cells_2) had no obvious characteristics of classic $B$ cell subtypes. We also detected three diverse subsets in macrophage populations and one subset of mixed macrophages and monocytes. Gene Ontology (GO) pathway enrichment showed that all these four clusters had increased expression of genes related to inflammatory responses, endocytosis, immune system process and chemotaxis, suggesting a possible role of these populations in inflammatory regulations (Fig. S3G-J).

Comparing the effects of HHcy with WD on ApoE ${ }^{-/-}$mice, cell composition analyses of our scRNA-seq data showed that, $\mathrm{B}$ cells appeared to be the most abundant population $(47.22 \%)$ in the vessels of $\mathrm{HHcy} \mathrm{ApoE}^{-/-}$mice, also showed 

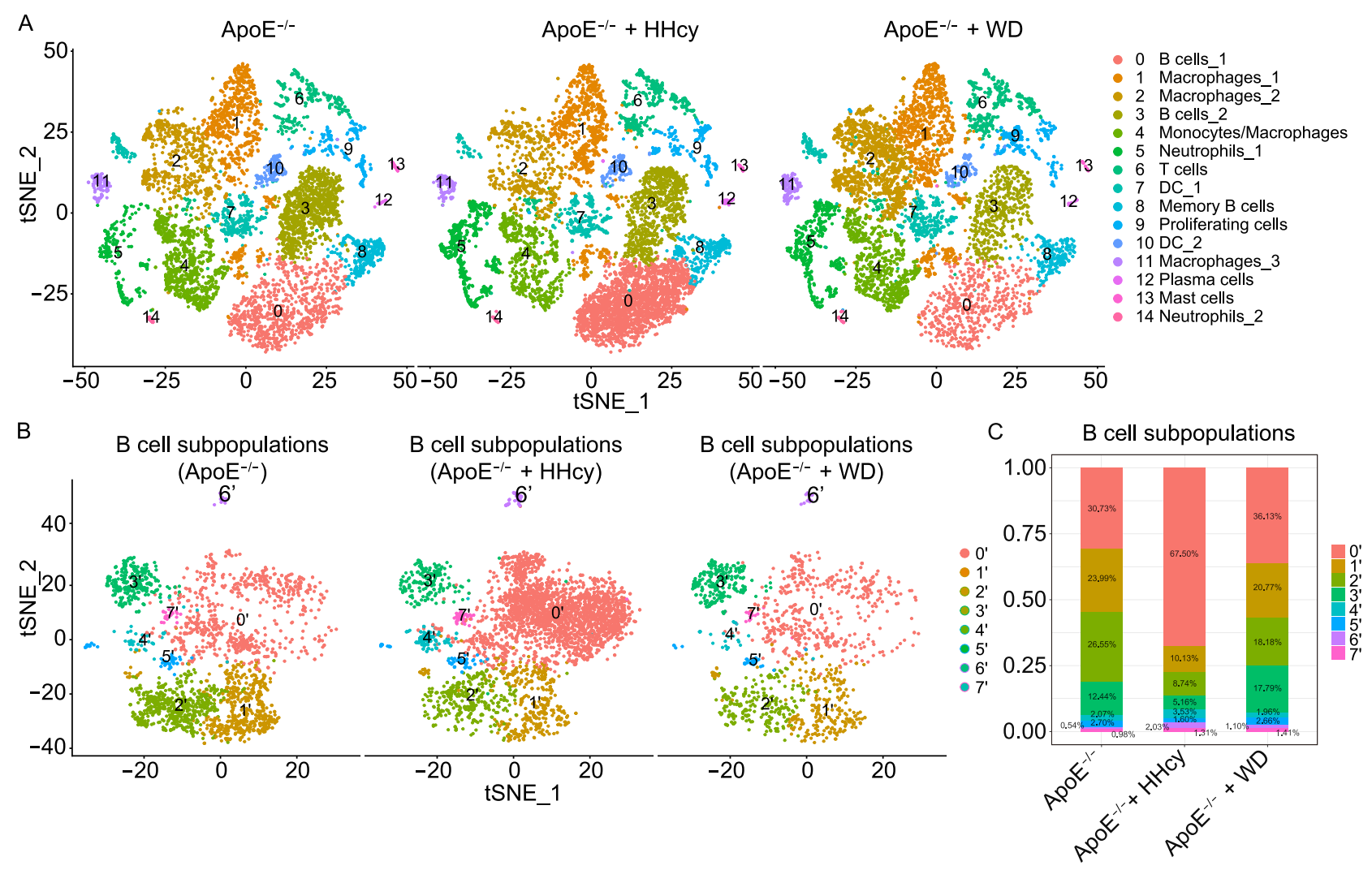

D Go enriment_B cells_Cluster0'_top10

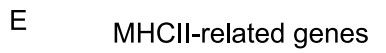

F
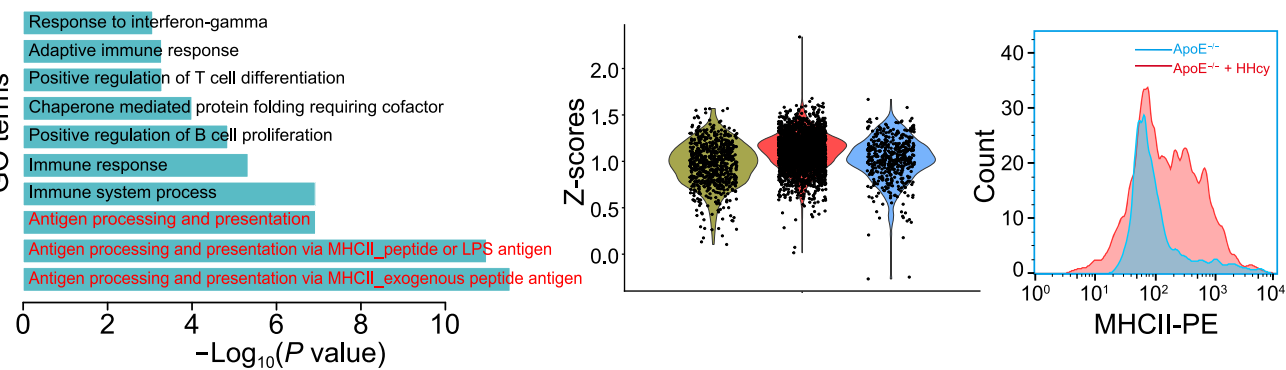

MHCII in B cells

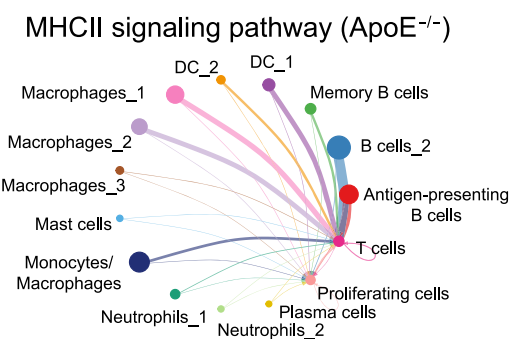

$\mathrm{H}$

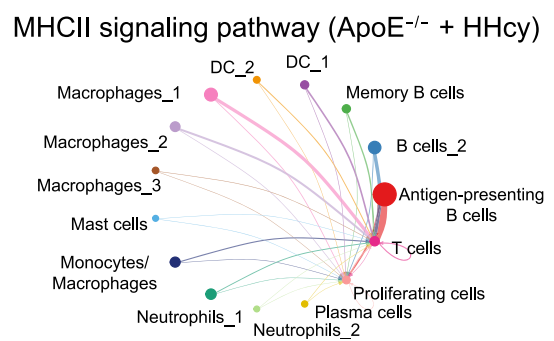

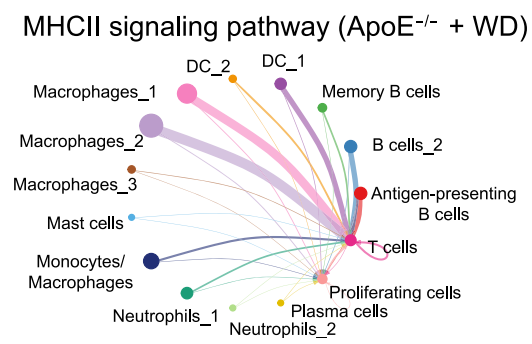

Figure 1. Antigen-presenting $B$ cells play a dominant role in antigen presentation in HHcy-accelerated atherosclerosis. (A) tSNE plots showing color-coded cell clusters in the three groups. (B) tSNE plots displaying eight distinct B cell clusters. (C) Bar charts showing the percentages of $B$ cell clusters in the three groups. (D) GO enrichment analyses of biological processes in cluster 0 '. (E) Violin plots showing the scaled expression scores (Z-scores) of MHCll-related genes $(H 2-A a, H 2-E b 1, H 2-A b 1, H 2-O b, H 2-$ Eb2, H2-Oa, H2-DMb2, Ctss, Cd74, H2-DMa, and Ctse) across the three groups. (F) Flow cytometry analyses showing the levels of $\mathrm{MHCll}$ in $\mathrm{CD} 19^{+} \mathrm{B}$ cells from atherosclerotic aortas. (G-l) Circle plots showing MHCIl signaling pathway in the three groups. Data represent mean \pm SEM $(n=4$ in $(\mathrm{F}))$. * $P<0.05$ by an unpaired 2-tailed $t$ test $(\mathrm{F})$. 
the most significant increase compared with $\mathrm{ApoE}^{-/-}$mice fed a $C D$, while macrophages/monocytes became the most abundant population in the WD ApoE ${ }^{-/}$group (Figs. $1 \mathrm{~A}$ and S4A). Of note, in the HHcy ApoE ${ }^{--}$group, a specific $B$ cell cluster (cluster 0, B cells_1) was dramatically increased to $30.25 \%\left(\mathrm{ApoE}^{-/-}: 13.65 \%\right.$, WD ApoE ${ }^{-/-}: 8.13 \%$ ) (Figs. $1 \mathrm{~A}$ and S4B). In addition, the proportion of neutrophils and $T$ cells was increased, and the proportion of DCs and mast cells was decreased in the $\mathrm{HHcy} \mathrm{ApoE}^{-/-}$group (Fig. S4A and S4B). Focused analysis on proliferating cells (cluster 9) revealed that this cluster contains $B$ cells, macrophages, monocytes, T cells and DCs (Fig. S4C and S4D). Among them, macrophages/monocytes became the majority of proliferating cells in the $\mathrm{WD} \mathrm{ApoE}^{-/-}$group, and $\mathrm{CD} 4^{+} / \mathrm{CD} 8^{+}$ $\mathrm{T}$ cells were highly increased in the $\mathrm{HHcy} \mathrm{ApoE}^{-/-}$group (Fig. S4E).

To confirm the findings from scRNA-seq, we further performed flow cytometry to detect the changes of immune cell $\left(\mathrm{CD}_{4} 5^{+}\right.$gated) proportions in the atherosclerotic aortas (Fig. S5A). Consistent with the scRNA-seq data, significant increases in the proportions of $\mathrm{B}$ cells and $\mathrm{CD} 3^{+} \mathrm{T}$ cells, as well as decreases in the proportions of macrophages in the HHcy ApoE ${ }^{--}$group were detected (Fig. S5B and S5C), compared with the $\mathrm{ApoE}^{-/-}$group. We also observed similar decreases in the proportions of $B$ cells, and the increases in the proportions of $T$ cells and macrophages in the WD $\mathrm{ApoE}^{-/-}$group (Fig. S5B-D). Collectively, our results present a comprehensive immune cell atlas in the aortas of HHcy $\mathrm{ApoE}^{-/-}$mice, and reveal distinct immune cell compositions from those in the aortas of WD-fed $\mathrm{ApoE}^{-/-}$mice.

To identify the possible function of $B$ cells, which were one of the most abundant and dynamic cell populations in this mouse model, focused analysis of $B$ cell populations was performed and identified eight $B$ cell subsets (Fig. 1B). Among them, the proportion of cluster 0' was dramatically increased from $30.73 \%$ to $67.50 \%$ in atherosclerotic aortas of the $\mathrm{HHcy} \mathrm{ApoE}^{-/-}$group compared with that of the $\mathrm{ApoE}^{-/-}$ group (Fig. 1C). Strikingly, GO pathway enrichment analyses showed that antigen presentation was the dominant pathways in cluster 0' (top 3) (Fig. 1D). MHCll-related genes, such as H2-Oa, H2-Eb2, H2-DMa (genes encoding the components of the MHCII complex) and Ciita (class II transactivator, the master transactivator for $\mathrm{MHCll}$ molecule expression) were highly expressed in cluster 0', compared with other B cell subpopulations (Fig. S6A-D). Average expression analyses by volcano plot revealed that cluster 0 ' showed substantial differences from other B cells in terms of gene expression profile. These $\mathrm{B}$ cells highly expressed MHCll-related genes H2-Aa, H2-Ab1, H2-Oa, H2-Ob, H2Eb2, Cd74 and Ciita (Fig. S6E). High expression of chemokine receptors Ccr6 and Cxcr4 was also observed in cluster 0' (Fig. S6E). These results indicate that MHCll-mediated antigen presentation might be one of the most important function in cluster 0 ', and migration from peripheral sites might be possible source of these B cells. B cells_1 (cluster 0 in Fig. $1 \mathrm{~A}$ ) was the majority of cluster 0 ' in focused analysis. Therefore, we annotated these $B$ cells (cluster 0 in Fig. $1 \mathrm{~A}$ or cluster 0 ' in Fig. $1 \mathrm{~B}$ ) as "antigen-presenting $\mathrm{B}$ cells".

Interestingly, inside this antigen-presenting B cell cluster, the expression of $\mathrm{MHCll}$-related genes was also highly upregulated in the $\mathrm{HHcy} \mathrm{ApoE}^{-/-}$group specifically (Figs. $1 \mathrm{E}$ and $\mathrm{S} 6 \mathrm{~F}-\mathrm{I})$. Flow cytometry further confirmed this finding in the protein level (Fig. 1F). In addition, activated $\mathrm{CD}^{+}{ }^{+} \mathrm{T}$ cells $\left(\mathrm{CD}^{+}{ }^{+} \mathrm{IFN}-\mathrm{Y}^{+}\right)$were also highly increased in the aortas from $\mathrm{HHcy} \mathrm{ApoE}{ }^{-/-}$mice (Fig. S6J). These results were consistent with the results from the scRNA-seq that the proportions of $T$ cells and proliferating $T$ cells were highly increased. Moreover, we performed focused analysis on cluster 6 in Fig. $1 \mathrm{~A}$ and revealed that $\mathrm{CD} 4^{+} \mathrm{T}$ cells were the most dynamic cluster with increases in the $\mathrm{HHcy} \mathrm{ApoE}^{-1-}$ group (Fig. S6K-M). Additionally, type 2 innate lymphoid cells (ILC2), natural killer (NK) cells and $y \delta T$ cells with small numbers were also identified in this cluster (Fig. S6K-M). Taken together, our results demonstrate increases in cell numbers and $\mathrm{MHCll}-$ related gene expression of antigenpresenting $\mathrm{B}$ cells in the $\mathrm{HHcy} \mathrm{ApoE}^{--}$group. The upregulation of $\mathrm{MHCll}$ signaling in $\mathrm{B}$ cells may further increase $\mathrm{CD}^{+} \mathrm{T}$ cell activation in HHcy-AA.

MHCll-mediated antigen presentation is a typical form of cell-cell contact between professional antigen presenting cells and $\mathrm{CD4}^{+} \mathrm{T}$ cells. To explore the potential intercellular communications among the identified immune cells and evaluate the role of antigen-presenting B cells, we performed cell-cell contact analysis using major immune cell groups via CellChat (Jin, et al., 2021). We identified both five outgoing and incoming communication patterns, and revealed $\mathrm{B}$ cells as a major source of MHCII signaling (outgoing pattern 2), while T cells served as a prominent receiver of this signaling (incoming pattern 2) in the $\mathrm{HHcy} \mathrm{ApoE}^{-/-}$group (Fig. S7AC). To further understand the role of MHCIl signaling in HHcy-AA, we analyzed MHCIl pathway networks among all finer immune cell clusters. While multiple macrophages, DCs and $B$ cell subsets displayed intermediate and strong communication with $\mathrm{T}$ cells in the control $\mathrm{ApoE}^{-/}$group and the WD $\mathrm{ApoE}^{-/-}$group, we observed a dominant interaction between antigen-presenting $B$ cells and T cells in the HHcy $\mathrm{ApoE}^{-/-}$group (Fig. 1G-I). These results suggest that antigen-presenting $B$ cells are the most dominant source of $\mathrm{MHCll}$ signaling in HHcy-AA.

MHCII-mediated antigen presentation between $B$ and $T$ cells requires the help of costimulatory molecules. We noticed that the inducible T-cell costimulator (ICOS) pathways was also enriched in the $\mathrm{HHcy} \mathrm{ApoE}^{-/-}$group (Fig. S7A-C). Similarly, antigen-presenting $B$ cells were revealed as an important source of the ICOS pathways in the $\mathrm{HHcy} \mathrm{ApoE}^{-/-}$group, but not in the other two groups (Fig. S7D-F). We also observed increases in the expression of MHCll-related genes $(\mathrm{H} 2-\mathrm{Oa})$ in antigen presenting $\mathrm{B}$ cells and the T cell receptor (TCR)-related gene $\mathrm{Cd} 4$ in $\mathrm{T}$ cells were highly upregulated (Fig. S7G and S7H). Costimulatory molecules (Icos/ in antigen-presenting B cells, Icos in 
T cells) were also elevated in the $\mathrm{HHcy} \mathrm{ApoE}^{-/-}$group (Fig. S7I and S7J). Flow cytometry showed the similar changes of MHCII and ICOSL in aortic B cells (Fig. S7K and S7L). In addition, plasma IFN-y and IL-2, which are mainly secreted from activated $T$ cells, were significantly increased in $\mathrm{HH}_{\text {cy }} \mathrm{ApoE}^{-/-}$mice (Fig. S7M and S7N). To further explore the localization of the increased $\mathrm{B}$ cells and $\mathrm{CD} 4^{+} \mathrm{T}$ cells, we performed confocal imaging analysis. The results showed that $\mathrm{B}$ cells and $\mathrm{CD} 4^{+}$T cells mainly localized at the adventitia of the vessels, and artery tertiary lymphoid organs (ATLOs) $\left(\mathrm{CD} 19^{+} \mathrm{CD} 4^{+} \mathrm{GL} 7^{+}\right)$were observed in the aortas from $\mathrm{HHcy} \mathrm{ApoE}^{-/-}$mice (Fig. S7O), but not in those from $\mathrm{ApoE}^{-/-}$mice and WD ApoE ${ }^{-/-}$mice (data not shown). Therefore, these results indicate that $\mathrm{MHCll}$ and costimulatory molecule signaling might be critical for the interaction between $B$ cells and T cells in the adventitia of HHcy-AA.

To elucidate the underlying mechanisms, splenic CD19+ $\mathrm{B}$ cells were isolated from $\mathrm{C} 57 \mathrm{BL} / 6 \mathrm{~J}$ mice and cultured with or without Hcy $(100 \mu \mathrm{mol} / \mathrm{L})$ for $24 \mathrm{~h}$, and then these cultured $B$ cells were subjected to transcriptome sequencing analysis. The results showed that the expression of MHCll-related genes, including genes encoding $\mathrm{MHCll}$ components and the upstream master regulator of MHCII expression, Ciita, was significantly upregulated by Hcy (Fig. 2A). Quantitative PCR validated these data (Fig. S8A). Flow cytometry and Western blot showed that Hcy significantly elevated the expression of MHCII and CIITA (Figs. 2B and S8B). The results from confocal images also showed an elevation in $\mathrm{MHCll}$ translocation to the plasma membrane of $\mathrm{B}$ cells (Fig. 2C). These data demonstrate that Hcy upregulates $\mathrm{MHCll}$ expression in $\mathrm{B}$ cells at both the gene and protein levels in vitro. To test the function of Hcy-increased $\mathrm{MHCll}$ in $B$ cells, we designed a B cell-T cell coculture system to measure the ability of MHCll-involved antigen processing and presentation in vitro (Fig. S8C), which is widely used (Deng, et al., 2013). B cells pretreated with Hcy significantly increased the secretion of IL-2 and IFN- $y$ from $\mathrm{CD}^{+}{ }^{+} \mathrm{T}$ cells (whose TCR has antigen specificity for chicken ovalbumin, OVA) under stimulation with OVA (Fig. S8D and $\mathrm{S} 8 \mathrm{E})$. In the coculture system, we also detected an increase in the costimulatory molecules $\mathrm{Cd} 40, \mathrm{Cd} 80$, and $\mathrm{Cd} 86$ in B cells (Fig. S8F), and Cd4Olg, Cd2, and Cd27 in $\mathrm{CD}^{+}{ }^{+} \mathrm{T}$ cells (Fig. S8G). However, Hcy had little effect on T cell activation mediated by DCs and macrophages in their coculture system (Fig. S8H-K), suggesting a specific effect of Hcy on B cells as functional antigen-presenting cells.

We next sought to explore possible mechanisms involved in the regulation of $\mathrm{MHCll}$ expression in B cells by Hcy. PKM2 is a key metabolic enzyme in glycolysis and also act as a protein kinase to regulate gene expression while translocating into the nucleus. We previously reported that Hcy increases the expression of PKM2 in B cells (Deng, et al., 2017). To elucidate whether PKM2 is involved in Hcyinduced $\mathrm{MHCll}$ expression in B cells, we employed B cellspecific PKM2-deficient (CD19cre-PKM2 $\left.2^{\mathrm{fl} / \mathrm{fl}}\right)$ mice and isolated splenic B cells. Protein expression of PKM2 was efficiently depleted in these B cells (Fig. S9A), whereas Cd19 in B cells and PKM2 in T cells and macrophages were not affected (Fig. S9B-D). As expected, B cell-specific PKM2 deficiency significantly reduced the Hcy-induced upregulation of $\mathrm{MHCll}$ expression (Figs. 2D and S9E). Confocal imaging analysis confirmed the decreases in $\mathrm{MHCll}$ on the plasma membrane (Fig. S9F). Consistently, PKM2 deficiency also reversed the upregulation of the mRNA levels of MHCII-related genes in the Hcy group (Fig. S9G). We next tested whether PKM2 depletion affects the function of antigen presentation. While control B cells pretreated with Hcy promoted IFN-y and IL-2 production by OT-II CD4 ${ }^{+}$T cells, PKM2 deficiency in B cells significantly restricted the activation of OT-II $\mathrm{CD}^{+} \mathrm{T}$ cells (Fig. 2E). Furthermore, the upregulation of $\mathrm{B}$ cell costimulatory molecules $(\mathrm{Cd40}, \mathrm{Cd} 86)$ was also inhibited by PKM2 deficiency (Fig. S9H). These results demonstrate that PKM2 is essential for Hcy-upregulated $\mathrm{MHCll}$ expression in B cells, and further B cell-mediated $\mathrm{CD} 4^{+} \mathrm{T}$ cell activation.

In addition, previous studies have established that dimeric and monomeric forms of PKM2 can translocate into nucleus and regulate gene expression directly. ScRNA-seq data showed no changes in glycolysis, the tricarboxylic acid (TCA) cycle and the pentose phosphate pathway which are regulated by $\mathrm{PKM} 2$, in aortic $B$ cells from atherosclerotic mice (Fig. S9I). Both glycolysis inhibitor 2-DG and PKM2 enzymatic inhibitor shikonin (SKN) had no effect on Hcyinduced $\mathrm{MHCll}$ expression (Fig. S9J). Therefore, we speculated that changes in the oligomeric state and the nonenzymatic nuclear function of PKM2 might regulate MHCIIrelated gene expression. To confirm this hypothesis, we detected the oligomerization form of PKM2 in B cells. The results showed that Hcy promoted the transformation of PKM2 from the tetramer and dimer to the monomer (Fig. 2F), and significantly increased the translocation of PKM2 proteins into the nucleus (Fig. 2G). Moreover, PKM2 activator TEPP-46 $(10 \mu \mathrm{mol} / \mathrm{L})$, which stabilizes the PKM2 tetramer and prevents the accumulation of its dimeric or monomeric forms and nuclear translocation, significantly downregulated the expression of MHCII in B cells (Fig. S9K). Collectively, these results indicate that $\mathrm{Hcy}$ promotes the accumulation of monomeric forms of PKM2 and subsequently PKM2 nuclear translocation may possibly participate in the regulation of MHCII-related gene expression, via a non-metabolic role in $B$ cells.

To elucidate how PKM2 regulates the gene expression of MHCII, we focused on CIITA that was highly upregulated by Hcy both in vivo and in vitro. CIITA is a master transactivator of $\mathrm{MHCll}$ expression and is definitely required for the transcription of MHCII-related genes. Previous studies have found three promoters located within an upstream region of the Ciita gene that control the transcription of Ciita in mice (Kobayashi, et al., 2012) (Fig. S10A). We detected the presence of transcripts induced by promoters III and IV (Ciita-plll and Ciita-pIV) but not transcripts induced by promoter I in B cells (Ciita-pl) (Fig. S10B). Of note, Hcy 
A MHCll-related genes (RNA-seq) B

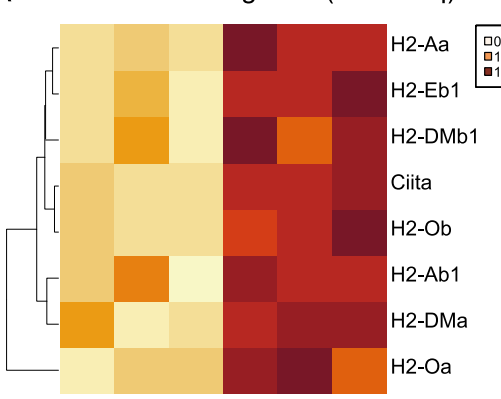

C
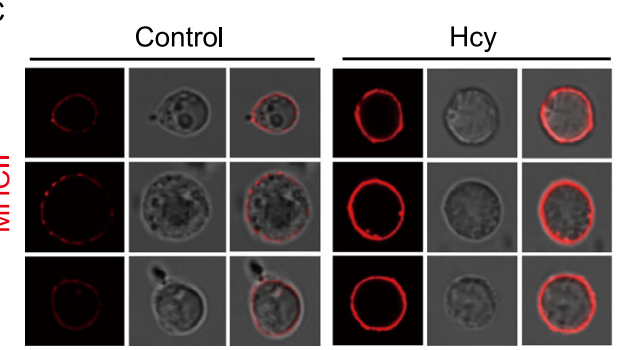

E IFN-Y IL-2
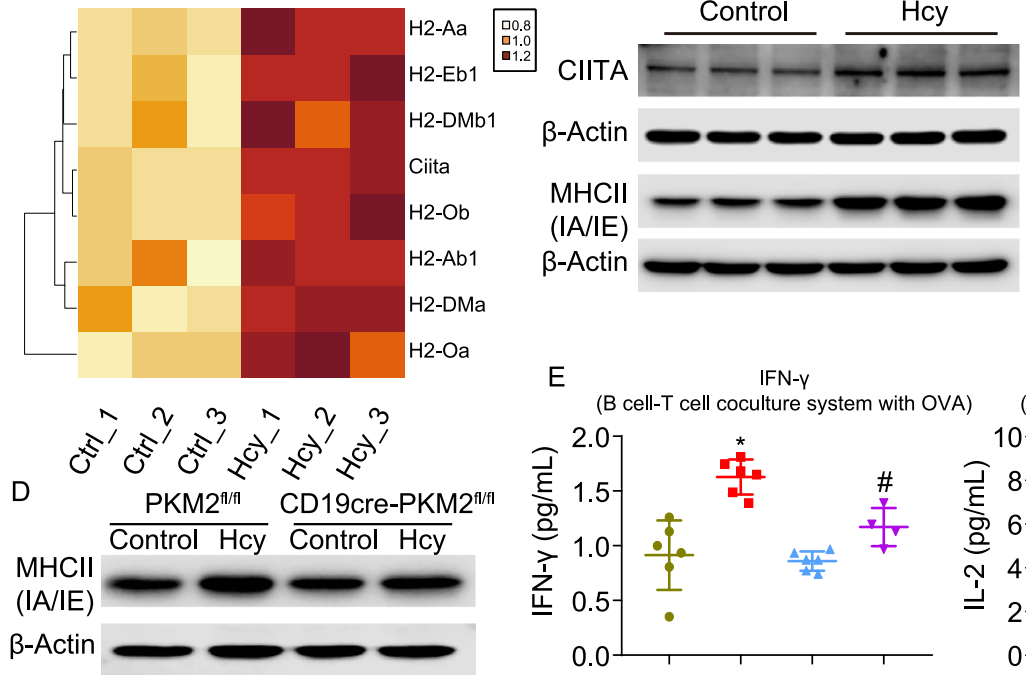

(B cell-T cell coculture system with OVA)

(B cell-T cell coculture system with OVA) $•$ Control PKM2 $2^{\text {intact }} B$ cells

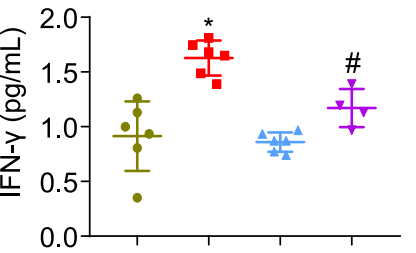

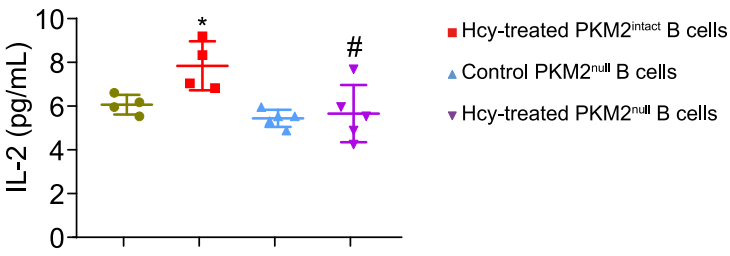

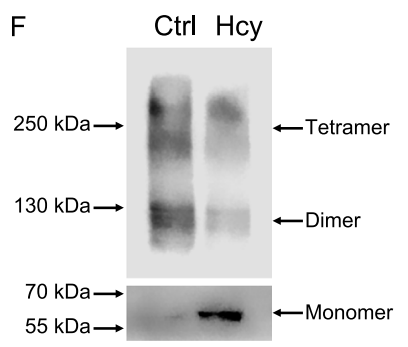

G

$\mathrm{H}$

Ciita-plll I Promoter III (binding with PKM2) $>$ (luciferase activity)
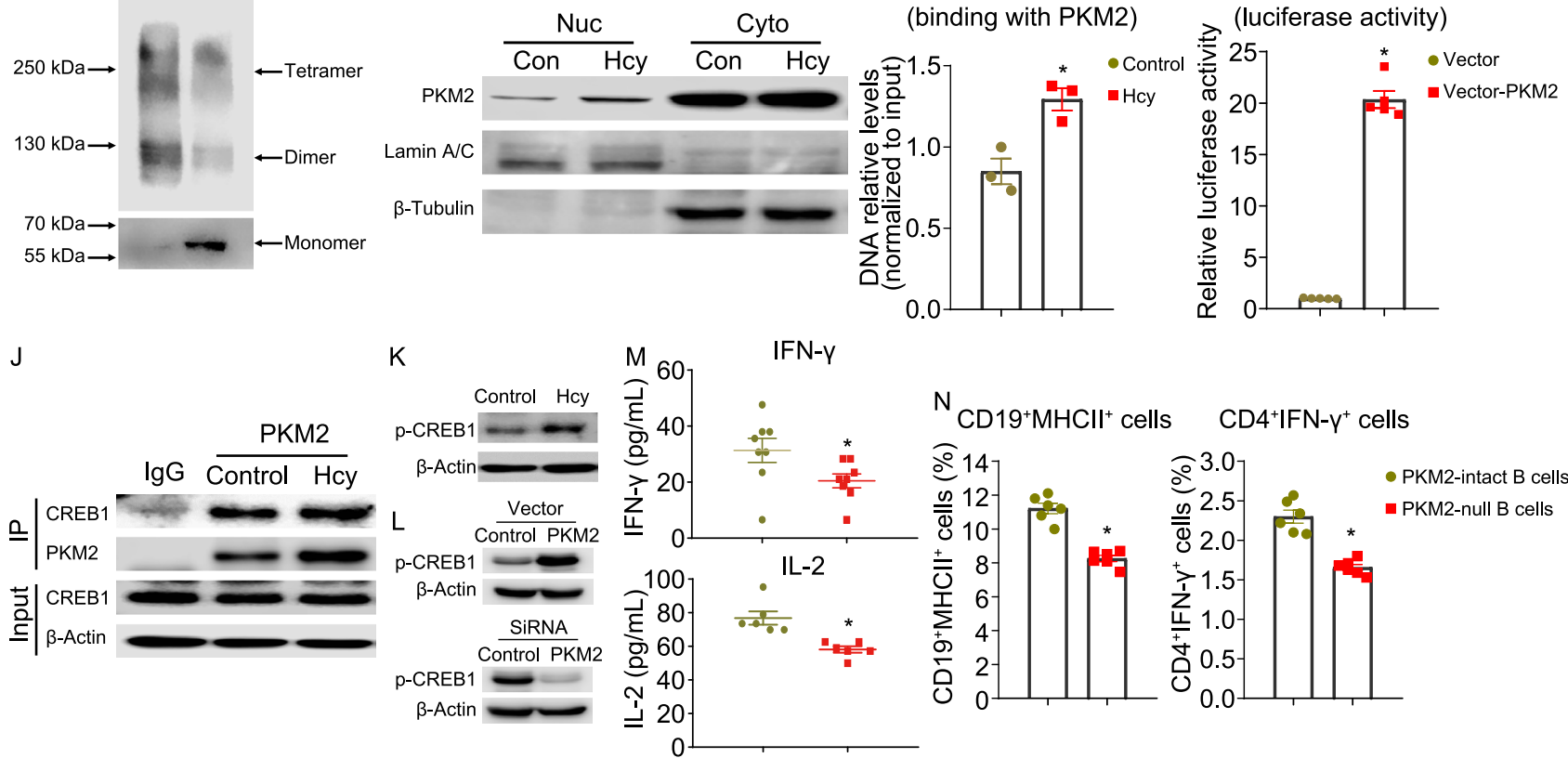

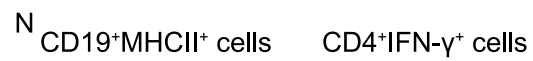

$\mathrm{O}$

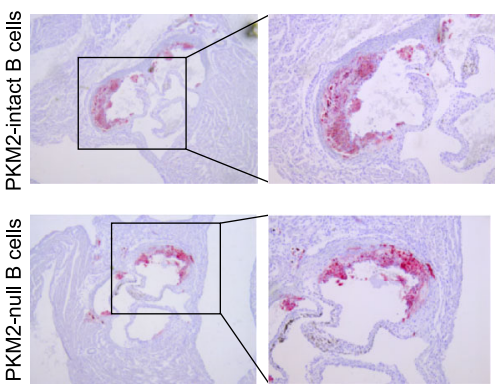

Plaque formation
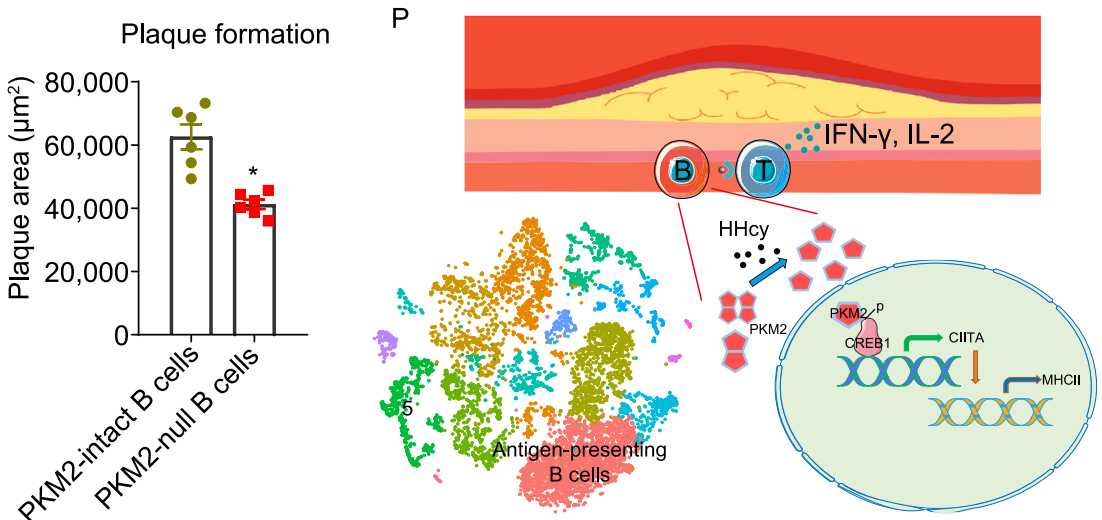
Figure 2. PKM2-induced antigen presentation is critical for $B$ cell-mediated $T$ cell activation in HHcy mice. (A-C) Purified $\mathrm{CD} 19^{+}$splenic $\mathrm{B}$ cells from $\mathrm{C} 57 \mathrm{BL} / 6 \mathrm{~J}$ mice were cultured with or without Hcy $(100 \mu \mathrm{mol} / \mathrm{L})$ for $24 \mathrm{~h}$. (A) Heatmap showing the mRNA levels of MHCll-related genes identified by RNA-seq. (B) The CIITA and MHCII expression and quantification were analyzed via Western blot. (C) Representative images showed staining of $\mathrm{MHCll}$ on B cells. (D) Purified CD19 ${ }^{+}$splenic $\mathrm{B}$ cells from $\mathrm{PKM} 2^{\mathrm{fl} / \mathrm{fl}}$ and $\mathrm{CD} 19 \mathrm{CrePKM} 2^{\mathrm{fl} / \mathrm{fl}}$ mice were cultured with or without Hcy $(100 \mu \mathrm{mol} / \mathrm{L})$ for $24 \mathrm{~h}$. MHCll protein expression was analyzed and quantified via Western blot. (E) Splenic B cells purified from PKM2 $2^{\mathrm{fl} / f l}$ and CD19CrePKM2 $2^{\mathrm{fl} / \mathrm{fl}}$ mice (showed as PKM2 ${ }^{\text {intact }}$ and PKM2 ${ }^{\text {null }} B$ cells, respectively) were pretreated with or without $100 \mu \mathrm{mol} / \mathrm{L}$ Hcy for $24 \mathrm{~h}$ and then cocultured with OVA-specific $\mathrm{CD}^{+} \mathrm{T}$ cells. OVA was added for another $48 \mathrm{~h}$. IFN-y and IL-2 secretion was analyzed via ELISA. (F-H) Purified CD $19^{+}$splenic B cells from C57BL/6J mice were cultured with or without Hcy $(100 \mu \mathrm{mol} / \mathrm{L})$ for $24 \mathrm{~h}$. (F) B cell lysates were cross-linked with glutaraldehyde and subjected to Western blot for detecting PKM2 oligomer states. (G) Nucleic and cytoplasmic proteins from B cells were extracted and subjected to Western blot for PKM2 detection. Lamin $A / C$ is a marker of nucleic proteins. $\beta$-Tubulin is a marker of cytoplasmic proteins. $(\mathrm{H})$ The DNA levels of PKM2-binding Ciita promoter III were measured via ChIP using an anti-PKM2 antibody and qPCR. (I) The transcriptional activity of Ciita promoter III with or without PKM2 overexpression was detected via the dual-luciferase system. (J) Purified CD19 ${ }^{+}$splenic B cells from C57BL/6J mice were cultured with or without Hcy (100 $\mu \mathrm{mol} / \mathrm{L})$ for $24 \mathrm{~h}$, and then cell extracts were subjected to coimmunoprecipitation with an antibody against PKM2. IgG servers as a negative control. Binding of PKM2 and CREB1 was detected and quantified via Western blot. (K) Purified $\mathrm{CD} 19^{+}$splenic $\mathrm{B}$ cells from C57BL/6J mice were cultured with or without Hcy $(100 \mu \mathrm{mol} / \mathrm{L})$ for $24 \mathrm{~h}$. Phosphorylation of CREB1 in Hcy-treated $B$ cells was detected via Western blot with an antibody against p-CREB1. (L) Phosphorylation of CREB1 in primary $\mathrm{B}$ cells with $\mathrm{Pkm} 2$ overexpression by plasmid transfection $(1.25 \mu \mathrm{g} / \mathrm{mL}, 48 \mathrm{~h}$ ) or Pkm2 knockdown by siRNA (50 pmol/ $\mathrm{mL}, 48 \mathrm{~h}$ ) was detected via Western blot. (M-O) Eight-week-old female $\mathrm{ApoE}^{-/-}$mice were injected with antibodies against CD19 and CD20 to deplete B cells. After 7 days, splenic B cells from $\mathrm{PKM} 2^{\mathrm{fl} / \mathrm{fl}}$ or $\mathrm{CD} 19 \mathrm{crePKM} 2^{\mathrm{fl} / \mathrm{fl}}$ mice were transferred into $\mathrm{B}$ cell-deficient $\mathrm{ApoE}^{-1-}$ mice, and drinking water supplemented with Hcy (1.8 g/L) was provided for 28 days. (M) Plasma IFN-y and IL-2 were analyzed via ELISA. (N) Flow cytometry analyses showing the percentages of $\mathrm{CD} 19^{+} \mathrm{MHCII}^{+}$cells and $\mathrm{CD} 4^{+} \mathrm{IFN}-$ $\mathrm{Y}^{+}$cells in atherosclerotic aortas. (O) Representative images showing cross-sections of aortic roots stained with Oil Red O, to assess and quantify plaque formation and lipid deposition. (P) Graphic model of this work. Antigen-presenting B cells appear to be the most abundant populations in immune cell atlas of HHcy-AA. Hcy increases the accumulation of PKM2 monomer and its nuclear translocation. Nuclear PKM2 interacts with and phosphorylates CREB1, induces CIITA-MHCII expression, and further increases $B$ cell- $T$ cell interaction, therefore activates $\mathrm{CD} 4^{+} \mathrm{T}$ cells. Data represent the mean $\pm \operatorname{SEM}(n=3$ in $(\mathrm{H}), n=4-6$ in (E), $n=6$ in ( $\mathrm{N}$ and $\mathrm{O}), n=6-8$ in $(\mathrm{M}))$. ${ }^{*} P<$ 0.05 by an unpaired 2-tailed $t$ test $(\mathrm{H}, \mathrm{I}, \mathrm{M}-\mathrm{O})$. ${ }^{*}$ or $\# P<0.05$ by one-way ANOVA followed by Tukey's test for multiple comparisons $(E)$. *Indicates the comparison with the Control group $(\mathrm{H})$, the vector group (I), or the PKM2 ${ }^{\text {intact }} \mathrm{B}$ cell group (M-O), \#Indicates the comparison with the Hcy-treated PKM2intact B cells group (E).

specifically increased the expression of Ciita-plll-induced transcripts (Fig. S10B). We next performed chromatin immunoprecipitation (chIP) assays and revealed the binding of PKM2 to Ciita-plll, while Hcy enhanced this binding process (Fig. 2H). A dual-luciferase (firefly and Renilla) reporter assay system revealed that PKM2 overexpression into HEK293T cells significantly promoted transcription induced by Ciita-pllI but not Ciita-pIV (Figs. 2I and S10C). In primary B cells, transfection of the PKM2 plasmid also significantly increased the CIITA at the mRNA and protein levels, as well as its downstream MHCll-related genes (Fig. S10D and S10E). Collectively, these results demonstrate that Hcy-increased PKM2 induces CIITA expression via binding to CiitaplII.

It remains unclear whether PKM2 binds to Ciita-plll directly or indirectly. Although previous reports did not discover any DNA binding domain or motif for PKM2, several transcription factors linking PKM2 and targeted genes have been suggested. We analyzed the relative scores of potential transcription factors targeting Ciita using ChIP-Atlas (Oki, et al., 2018), and revealed interferon regulatory factor 4 (IRF4) and cyclic AMP-responsive element-binding protein 1 (CREB1) as the top 2 candidates (Fig. S10F), although neither of these proteins have been reported to interact with PKM2. We next tested whether IRF4 and CREB1 were involved in these processes. The results from co-IP assays showed that PKM2 directly bound to CREB1, which was significantly promoted by Hcy (Fig. 2J). However, the binding of IRF4 and PKM2 was not detected (data not shown). CREB1 is an important regulator of Ciita and can directly bind to Ciita-plll (van der Stoep, et al., 2002), and the activity of CREB1 as a transcription factor depends on its phosphorylation state (Altarejos, et al., 2011). Of note, the upregulation of CREB1 phosphorylation in B cells by Hcy was observed (Fig. 2K), consistent with a previous report that Hcy induces CREB phosphorylation in hepatocytes (HepG2) (Woo, et al., 2006). More importantly, in the scRNAseq data, several target genes of CREB1 (Ap2b1, Eif2b1, Noc4l, and Haus5) were highly upregulated in antigen-presenting $\mathrm{B}$ cells in the HHcy ApoE $\mathrm{H}^{-/-}$group, however, they were not affected by WD (data not shown). PKM2 knockdown and overexpression also dramatically downregulated and upregulated the phosphorylation of CREB1, respectively (Fig. 2L). Taken together, these results reveal a novel pathway that Hcy-increased nuclear PKM2 directly binds to CREB1, phosphorylates CREB1, and further PKM2-CREB1 
complex binds to CIITA promoters and induces CIITA and its downstream $\mathrm{MHCll}$ expression in $\mathrm{B}$ cells.

To further elucidate the role of PKM2 in regulating antigen presentation in vivo, we first used an OT-II mouse model to evaluate the antigen presentation process between $B$ and $T$ cells (Fig. S10G). We applied anti-CD19 and anti-CD20 monoclonal antibodies to deplete B cells in OT-II mice for 7 days. B cells were efficiently depleted in the peripheral blood (Fig. S10H), without affecting the other professional antigen presenting cells (DCs and F4/80 ${ }^{+}$cells) (Fig. S10I). PKM2intact or PKM2-null B cells were then transferred into these recipient mice. At the same time, OVA was injected, and Hcy-containing drinking water was provided for another 14 days. Results showed the expression of MHCll-related genes was lower in PKM2-null B cells (Fig. S10J). ELISA and flow cytometry showed that mice PKM2-null B cell transferring lowered $\mathrm{T}$ cell activation (Figs. $2 \mathrm{M}, \mathrm{S} 10 \mathrm{~K}$ and $\mathrm{S} 10 \mathrm{~L})$. The costimulatory molecules $\mathrm{Cd40}, \mathrm{Cd} 80$, and Cd86 in splenic B cells and Cd40lg, Cd27, and Cd2 in splenic $\mathrm{CD}^{+}{ }^{+}$T cells from recipient mice with $\mathrm{PKM} 2-$ null $\mathrm{B}$ cells were also downregulated (Fig. S10M and S10N). These data confirmed an essential role of PKM2 of $\mathrm{B}$ cells in regulating $\mathrm{CD}^{+}{ }^{+} \mathrm{T}$ cell activation in vivo.

To further confirm the role of B cell PKM2 in HHcy-accelerated atherosclerosis, we applied the same adoptive transferring strategy in the early stage atherosclerosis mouse model, in which $\mathrm{ApoE}^{-/-}$mice were supplemented with or without Hcy-containing water for 28 days. Flow cytometry results showed that PKM2-null B cell transferring lowered the numbers of $B$ cells and $T$ cells in $\mathrm{HHcy}-\mathrm{AA}$, but the numbers of macrophages were slightly increased (Fig. S11A-D). Further analysis revealed that the ability of $B$ cell antigen presentation $\left(\mathrm{CD} 19^{+} \mathrm{MHCl}^{+}\right)$and activation of $\mathrm{CD}^{+}{ }^{+}$cells $\left(\mathrm{CD} 4^{+} \mathrm{IFN}-\mathrm{\gamma}^{+}\right)$was significantly decreased in the PKM2-null B cell transferring group (Fig. 2N). More importantly, Oil Red $O$ staining showed significant decrease in plaque formation by PKM2-null B cell transferring (Fig. 2O). Summarily, these data demonstrate that nuclear PKM2 is critical for $\mathrm{B}$ cells to activate $\mathrm{CD}^{+} \mathrm{T}$ cells, and mediates $\mathrm{HHcy}$-accelerated atherosclerosis, at least in part by inducing $\mathrm{MHCll}$ expression in $\mathrm{B}$ cells (Fig. 2P).

While we provided a comprehensive immune cell atlas in HHcy-AA and highlighted the role of antigen presentation in local B cells, the limitations of our study should be acknowledged. First, the proportions of B cells are different between our study and a previous study (Winkels, et al., 2018), which might be due to the background, ages of the mouse, the time of specific-diet feeding, and the maintaining environment. The estimation of immune cells in different models following the same procedure is necessary. Second, potential antigens presented by antigen-presenting $B$ cells in HHcy-AA are not identified yet.

In summary, we first provide a comprehensive immune cell landscape in HHcy-AA and reveal that aortic $B$ cells mainly function as antigen-presenting cells with dominance in atherosclerosis, especially those accelerated by HHcy.
Moreover, the role of nuclear PKM2-CREB1-CIITA axis in $\mathrm{Hcy}$-induced $\mathrm{MHCll}$ expression in B cells was firstly clarified. This finding might provide new insights into HHcy-accelerated atherosclerosis and novel targets for the prevention and treatment of this disease.

\section{FOOTNOTES}

Xiaolong Ma, Jiacheng Deng, Lulu Han, Yuwei Song, Yutong Miao, Xing Du, Guohui Dang, DongminYang, Bitao Zhong, Changtao Jiang, Wei Kong, Qingbo Xu, Juan Feng, Xian Wang.

Xiaolong Ma designed the study, performed the experiments, analyzed the data, and wrote the paper. Jiacheng Deng, Lulu Han, Yuwei Song, Yutong Miao, Xing Du, Guohui Dang, Dongmin Yang, and Bitao Zhong provided important help. Changtao Jiang, Wei Kong, and Qingbo Xu provided guidance during the study. Juan Feng and Xian Wang designed the study, reviewed and made important suggestions to the manuscript. All authors read and approved the final manuscript.

We also thank Prof. Xiaoyan Qiu for great advice.

None of the authors has conflict of interests to report.

This work was supported by grants from National Natural Science Foundation of China (31872787, 91739303, 91939105, 81770445,81921001 , and 82170476), and Natural Science Foundation of Beijing, China (No. M21008).

The manuscript does not contain clinical studies or patient data. All institutional and national guidelines for the care and use of laboratory animals were followed.

ScRNAseq data of our study are available in Gene Expression Omnibus (GSE190220).

Xiaolong Ma ${ }^{1}$ (D), Jiacheng Deng ${ }^{2}$ (D), Lulu Han ${ }^{1}$ (D), Yuwei Song ${ }^{1}$ (D), Yutong Miao ${ }^{3}$ (D), Xing $\mathrm{Du}^{1}$ (D), Guohui Dang ${ }^{1}$ (D), Dongmin Yang ${ }^{1}$ (D), Bitao Zhong ${ }^{1}$ (D), Changtao Jiang ${ }^{1}$ (D), Wei Kong ${ }^{1}$ (D), Qingbo $\mathrm{Xu}^{4}$ (D), Juan Feng ${ }^{1 凶}$ (D) Xian Wang ${ }^{1 凶}$ (D)

${ }^{1}$ Department of Physiology and Pathophysiology, School of Basic Medical Sciences, Key Laboratory of Molecular Cardiovascular Science, Ministry of Education, Peking University, Beijing 100191, China

${ }^{2}$ Cardiovascular Division, BHF Center of Vascular Regeneration, King's College London, London, UK

${ }^{3}$ Department of Clinical Laboratory, Beijing Chao-Yang Hospital, Capital Medical University, Beijing 100020, China

${ }^{4}$ Department of Cardiology, The First Affiliated Hospital, Zhejiang University, Hangzhou 310003, China

$\triangle$ Correspondence: juanfeng@bjmu.edu.cn (J. Feng), xwang@bjmu.edu.cn (X. Wang)

\section{OPEN ACCESS}

This article is licensed under a Creative Commons Attribution 4.0 International License, which permits use, sharing, adaptation, distribution and reproduction in any medium or format, as long as you give appropriate credit to the original author(s) and the source, provide a link to the Creative Commons licence, and indicate if 
changes were made. The images or other third party material in this article are included in the article's Creative Commons licence, unless indicated otherwise in a credit line to the material. If material is not included in the article's Creative Commons licence and your intended use is not permitted by statutory regulation or exceeds the permitted use, you will need to obtain permission directly from the copyright holder. To view a copy of this licence, visit http:// creativecommons.org/licenses/by/4.0/.

\section{REFERENCES}

Altarejos JY, Montminy M (2011) CREB and the CRTC co-activators: sensors for hormonal and metabolic signals. Nat Rev Mol Cell Biol 12:141-151

Dai J, Li W, Chang L, Zhang Z, Tang C, Wang N, Zhu Y, Wang X (2006) Role of redox factor-1 in hyperhomocysteinemia-accelerated atherosclerosis. Free Radic Biol Med 41:1566-1577

Deng T, Lyon CJ, Minze LJ, Lin J, Zou J, Liu JZ, Ren Y, Yin Z, Hamilton DJ, Reardon PR et al (2013) Class II major histocompatibility complex plays an essential role in obesity-induced adipose inflammation. Cell Metab 17:411-422

Deng J, Lu S, Liu H, Liu B, Jiang C, Xu Q, Feng J, Wang X (2017) Homocysteine activates $B$ cells via regulating PKM2-dependent metabolic reprogramming. J Immunol 198:170-183

Huo Y, Li J, Qin X, Huang Y, Wang X, Gottesman RF, Tang G, Wang $B$, Chen D, He M et al (2015) Efficacy of folic acid therapy in primary prevention of stroke among adults with hypertension in China: the CSPPT randomized clinical trial. JAMA 313:13251335

Jin S, Guerrero-Juarez CF, Zhang L, Chang I, Ramos R, Kuan CH, Myung P, Plikus MV, Nie Q (2021) Inference and analysis of cellcell communication using Cell Chat. Nat Commun 12:1088

Kobayashi KS, van den Elsen PJ (2012) NLRC5: a key regulator of MHC class I-dependent immune responses. Nat Rev Immunol 12:813-820
Luo Y, Feng J, Xu Q, Wang W, Wang X (2016) NSun2 Deficiency protects endothelium from inflammation via mRNA methylation of ICAM-1. Circ Res 118:944-956

Oki S, Ohta T, Shioi G, Hatanaka H, Ogasawara O, Okuda Y, Kawaji H, Nakaki R, Sese J, Meno C (2018) ChIP-Atlas: a data-mining suite powered by full integration of public ChIP-seq data. EMBO Rep 19

Stuart T, Butler A, Hoffman P, Hafemeister C, Papalexi E, Mauck WM, 3rd, Hao Y, Stoeckius M, Smibert P, Satija R (2019) Comprehensive Integration of Single-Cell Data. Cell 177:18881902 e 1821

Truelsen T, Krarup LH, Iversen HK, Mensah GA, Feigin VL, Sposato LA, Naghavi M (2015) Causes of death data in the global burden of disease estimates for ischemic and hemorrhagic stroke. Neuroepidemiology 45:152-160

van der Stoep N, Quinten E, van den Elsen PJ (2002) Transcriptional regulation of the $\mathrm{MHC}$ class II trans-activator (CIITA) promoter III: identification of a novel regulatory region in the 5'untranslated region and an important role for CAMP-responsive element binding protein 1 and activating transcription factor- 1 in CIITA-promoter III transcriptional activation in B lymphocytes. J Immunol 169:5061-5071

Weber G, Camagni G (1950) Frequency and entity of aortic atherosclerosis in chronic and acute endocarditis; inflammatory genesis of the atherosclerotic process. Arch De Vecchi Anat Patol 14:45-69

Winkels $\mathrm{H}$, Ehinger E, Vassallo M, Buscher K, Dinh HQ, Kobiyama $K$, Hamers AAJ, Cochain C, Vafadarnejad E, Saliba AE et al (2018) Atlas of the immune cell repertoire in mouse atherosclerosis defined by single-cell RNA-sequencing and mass cytometry. Circ Res 122:1675-1688

Woo CW, Siow YL, O K, (2006) Homocysteine activates cAMPresponse element binding protein in HepG2 through cAMP/PKA signaling pathway. Arterioscler Thromb Vasc Biol 26:1043-1050

Supplementary Information The online version contains supplementary material available at https://doi.org/10.1007/s13238-02100904-0. 